\title{
Designs, Synthesis, Docking Studies, and Biological Evaluation of Novel Berberine Derivatives Targeting Zika Virus
}

\author{
Cuong Quoc Nguyen, ${ }^{1}$ Thi Hong Minh Nguyen, ${ }^{2}$ Thi Thu Thuy Nguyen, ${ }^{3}$ Thi Buu Hue Bui, ${ }^{1}$ \\ Trong Tuan Nguyen, ${ }^{1}$ Nhu Thao Huynh, ${ }^{1}$ Thanh Do Le, ${ }^{3}$ Thi Mai Phuong Nguyen, ${ }^{4,5}$ \\ Duy Tuan Nguyen, ${ }^{6}$ Minh Tam Nguyen, ${ }^{7,8}$ Minh Quan Pham $\mathbb{D}^{4,9}$ Quang De Tran $\mathbb{D}^{1},{ }^{1}$ \\ and Hong Phuong Nguyen (iD) \\ ${ }^{1}$ Department of Chemistry, College of Natural Sciences, Can Tho University, Can Tho, Vietnam \\ ${ }^{2}$ Department of Life Science, University of Science and Technology of Ha Noi, Vietnam Academy of Science and Technology, \\ Ha Noi, Vietnam \\ ${ }^{3}$ Institute for Global Health Innovations, Duy Tan University, Da Nang, Vietnam \\ ${ }^{4}$ Graduate University of Science and Technology, Vietnam Academy of Science and Technology, Ha Noi, Vietnam \\ ${ }^{5}$ Institute of Biotechnology, Vietnam Academy of Science and Technology, Ha Noi, Vietnam \\ ${ }^{6}$ Faculty of Medicine, Nam Can Tho University, Can Tho, Vietnam \\ ${ }^{7}$ Computational Chemistry Research Group, Ton Duc Thang University, Ho Chi Minh, Vietnam \\ ${ }^{8}$ Faculty of Applied Sciences, Ton Duc Thang University, Ho Chi Minh, Vietnam \\ ${ }^{9}$ Institute of Natural Products Chemistry, Vietnam Academy of Science and Technology, Ha Noi, Vietnam \\ ${ }^{10}$ Institute of Research and Development, Duy Tan University, Da Nang, Vietnam
}

Correspondence should be addressed to Minh Quan Pham; minhquanaries@gmail.com, Quang De Tran; tqde@ctu.edu.vn, and Hong Phuong Nguyen; nguyenhongphuong@duytan.edu.vn

Received 18 January 2021; Revised 13 March 2021; Accepted 1 April 2021; Published 19 April 2021

Academic Editor: Ajaya Kumar Singh

Copyright () 2021 Cuong Quoc Nguyen et al. This is an open access article distributed under the Creative Commons Attribution License, which permits unrestricted use, distribution, and reproduction in any medium, provided the original work is properly cited.

The World Health Organization has designated Zika virus (ZIKV) as a dangerous, mosquito-borne flaviviral pathogen that was recently found to be responsible for a dramatically increased number of microcephaly cases and other congenital abnormalities in fetuses and newborns. There is neither a vaccine to prevent nor a drug to treat ZIKA virus infections, at the present time. Berberine (BBR) is a promising drug approved by FDA against flaviviral dengue virus, and BBR derivatives are of great interest in antiviral drug development. In this study, we synthesized eight BBR derivatives by introducing benzyl groups at the C-13 position of BBR and converting to respective 8-oxoberberine derivatives, performed molecular docking analysis, and evaluated their anti-Zika virus activity utilizing a cell-based phenotypic assay. Binding mode analysis, absolute binding free energy calculation, and structure-activity relationship studies of these compounds with ZIKV NS3 receptor were collected. Amongst these studied compounds, compound 4d with a structure of 13-(2,6-difluoro)-benzylberberine showed high binding affinity (docking score of $-7.31 \mathrm{kcal} / \mathrm{mol}$ ) towards ZIKV NS2B-NS3 protease with critical binding formed within the active site. In the cell-based assay, compound 4d displayed the highest antiviral efficacy against ZIKV with a selective index (SI) of 15.3, with 3.7-fold greater than that of berberine. Together, our study suggests that the potential ZIKV NS2B-NS3 protease inhibitor, compound 4d, is the best alternative to $\mathrm{BBR}$ and, further, extends an assuring platform for developing antiviral competitive inhibitors against ZIKV infection. 


\section{Introduction}

Zika virus (ZIKV) was noticed as a major health risk by the World Health Organization. ZIKV is a mosquito-borne pathogen family having a positive-sense single-stranded RNA genome, belonging to Flaviviridae family including dengue, West Nile, and Japanese encephalitis viruses. Unlike its mosquito-borne relatives, ZIKV has emerged from obscurity by its association with a suspected "congenital Zika syndrome," while causing asymptomatic or mild exanthematous febrile infections which are dengue or rubella-like in infected individuals [1]. ZIKV is thought to cause birth defects as well as neurological problems $[2,3]$. Additionally, ZIKV is associated with neural-inflammatory diseases such as Guillain-Barré syndrome and ophthalmological complications in adults [1]. Currently, there are no medicines or vaccines against ZIKV or specific antiviral treatment for clinical ZIKV infection. To address this medical need, novel ZIKV interventions suitable for prevention and therapeutic purposes are urgent.

Berberine (BBR), an active isoquinoline alkaloid extracted from the Chinese herb Coptis chinensis [4], has been widely used for the treatment of gastrointestinal infection and diarrhea [5]. Currently, BBR is a FDA-approved drug to be effective against flaviviral dengue virus [6]. Recent studies revealed that BBR has various pharmacological activities such as antimicrobial [7], antidiabetic [8], antihyperlipidemic action [9], antitumor [10], antiinflammatory [11], and antiparasitic [12]. Besides, several novel strategies have been developed for improving the bioavailability of BBR, which alternatively enhance its activity. Modifications of BBR structure is an effective pathway to yield derivatives with more valuable pharmacological properties.. Therefore, a series of BBR derivatives have been designed by transforming and modifying its chemical structure [13-15].

In that respect, novel BBR-derived compounds (4a-d and 5a-d) were designed and synthesized aiming at obtaining drug leads possibly exhibiting ameliorated antiZIKV properties. An unprecedented feature of this new class of BBR derivatives is the presence of heteroaromatic moieties bonded to the 13-position of the parent alkaloid skeleton through a linker and functionality, in a fashion to possibly create a geometric propensity for additional noncovalent aromatic interactions on the cellular target site [16]. The ZIKA inhibitory effect of BBR and eight selected BBR derivatives then performed molecular docking simulation and biological evaluation targeted ZIKV to find specific antiviral BBR derivative compounds that may be potential in the treatment of Zika.

\section{Materials and Methods}

\subsection{Chemistry}

2.1.1. General Information. All reagents were purchased from commercial sources and were used without further purification. Reactions were monitored by thin-layer chromatography on $0.2 \mathrm{~mm}$ precoated silica gel 60 F254 plates (Merck). Derivatives were synthesized in the laboratory and then were purified by flash column chromatography using silica gel 45-63 $\mu \mathrm{m}$ (230-400 mesh), $60 \AA$ pore size. ${ }^{1} \mathrm{H}$ NMR spectra were recorded on a Varian Mercury spectrophotometer (Agilent Technologies, Inc., CA, USA) at $300 \mathrm{MHz}$. Chemical shift values $(\delta)$ are given in parts per million (p.p.m) downfield from tetramethylsilane as an internal reference; coupling constants are given in hertz $(\mathrm{Hz})$ and spin multiplicities are given as $\mathrm{s}$ (singlet), d (doublet), dd (doublet of doublet), $\mathrm{t}$ (triplet), $\mathrm{q}$ (quartet), or m (multiplet).

(13-Benzylberberine) derivatives $\mathbf{4 a - b}$ and $\mathbf{5} \mathbf{a}-\mathbf{b}$ were prepared adopting the previously reported methods as shown in Figure 1 [17].

2.1.2. General Procedure for the Synthesis of Compound 2. Compound 1 (berberine chloride, $10.0 \mathrm{~g}, 26.9 \mathrm{mmol}$ ) was added to $5.0 \mathrm{M} \mathrm{NaOH}$ solution $(9.2 \mathrm{~g}$ of $\mathrm{NaOH}, 46 \mathrm{~mL}$ of $\mathrm{H}_{2} \mathrm{O}$ ) in a $250 \mathrm{~mL}$ size round-bottom flask at $0^{\circ} \mathrm{C}$. The mixture was stirred for $30 \mathrm{~min}$ before adding acetone $(10 \mathrm{~mL}$, $134.5 \mathrm{mmol}, 5.0 \mathrm{eq})$ dropwise at $0^{\circ} \mathrm{C}$. The mixture was stirred for $2 \mathrm{~h}$ at room temperature. Then, the solid was filtered and washed with $\mathrm{H}_{2} \mathrm{O}(80 \mathrm{~mL})$ and cold $80 \% \mathrm{MeOH}$ in $\mathrm{H}_{2} \mathrm{O}$ $(80 \mathrm{~mL})$ and dried under the reduced pressure system giving the desired product 2 as a brownish-yellow solid ( $8.4 \mathrm{~g})$ with $80 \%$ yield.

2.1.3. General Procedure for the Synthesis of Compound 4. Compound 2 (1.0 g, $2.96 \mathrm{mmol}, 1.0 \mathrm{eq})$ in $\mathrm{MeCN}$ (dried, $25 \mathrm{~mL}$ ) was added with various benzyl halides $3(5.93 \mathrm{mmol}$, $2.0 \mathrm{eq})$ and $\mathrm{NaI}(0.534 \mathrm{~g}, 3.55 \mathrm{mmol}, 1.2 \mathrm{eq})$ and stirred at $80^{\circ} \mathrm{C}$. After $4 \mathrm{~h}$, the reaction mixture was filtered, washed with MC 3 times. While the solid (BBR, recovered) was dried, the filtrate was extracted with $\mathrm{MC}$, dried with $\mathrm{Na}_{2} \mathrm{SO}_{4}$, filtered, concentrated, and purified by flash column chromatography (MC:MeOH, 20:1 to 10:1), which gave the desired product 4 as yellow solid.

2.1.4. General Procedure for the Synthesis of Compound 5. A stirred suspension of compound $4(300 \mathrm{mg})$ in $20 \% \mathrm{KOH}$ (aq, $20 \mathrm{~mL})$ was refluxed $\left(115^{\circ} \mathrm{C}\right)$ for $24 \mathrm{~h}$. After being cooled down to room temperature, the reaction mixture was extracted with MC twice. Then, combined organic phase was dried over $\mathrm{Na}_{2} \mathrm{SO}_{4}$, filtered, evaporated, and purified using flash column chromatography (EtOAc: Hex, $1: 1$ to $2: 1,3$ : 1 , and $4: 1$ ), which gave the desired product 5 as pale yellow solid.

\subsection{Molecular Docking Studies}

2.2.1. Ligands and Receptor. The three-dimensional structures of BBR and BBR derivatives (4a-d and 5a-d) were prepared using ChemDraw version 16.0 (PerkinElmer ${ }^{\circledR}$ ). The energy minimization was carried out using MM2 force field and quantum chemical calculations were performed by PM6 semiempirical method implemented in Gaussian 09 $[18,19]$. 


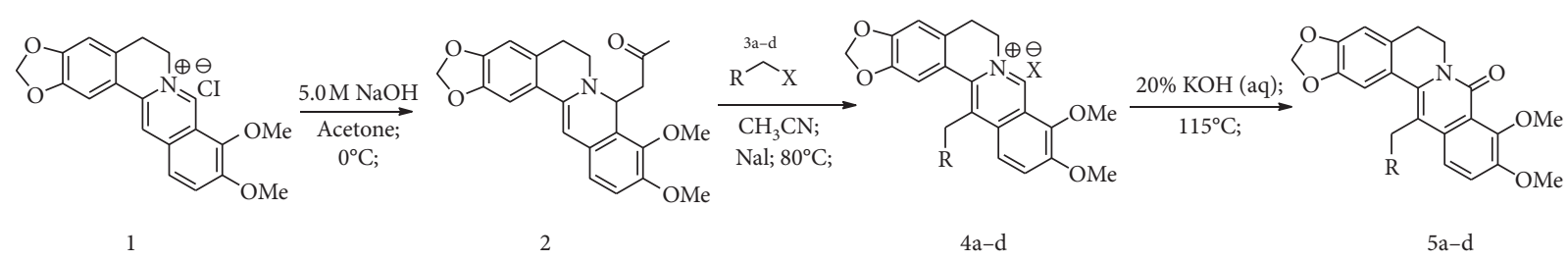

FIgURe 1: Synthesis protocol of BBR derivatives (13-benzylberberine) $\mathbf{4 a - d}$ and $\mathbf{5 a - d}$.

2.2.2. Docking Using AutoDock. The Graphical User Interface program named AutoDock Tools 1.5.6 (MGLTools) was employed to set up for docking simulations. The protein structure was prepared in order to obtain the correct ionization and tautomeric states of amino acid residues. Firstly, the heteroatoms including water molecules were removed and polar hydrogen atoms were added. Then, the Kollman charges and solvation parameters were assigned and Gasteiger charges were added to each atom. MGLTools assign the rigid roots to the ligands automatically and all other bonds were allowed to be rotatable.

The grid map of various atoms of the ligands and receptor was generated by AutoGrid. It was previously reported that His51, Asp75, and Ser135 play an important role in the functioning activity of NS2B-NS3 protease [20, 21]; thus, in the docking procedure, the grid box was chosen such that it incorporates these key residues. The energy scoring grid was prepared in $x \times y \times z$ directions $(80 \times 80 \times 80)$ with a grid spacing of $0.375 \AA$. During the docking process, the parameters of the Lamarckian genetic algorithm were as follows: 100 runs, elitism of 1 ; the mutation rate of 0.02 ; the population size of 150; a crossover rate of 0.80 ; number of generations of 27,000; the energy evaluations of 25,000,000; and the root-mean-square (RMS) cluster tolerance set to 2.0 $\AA$ in each run. The outputs from AutoDock 4.2.6 modeling studies were analyzed using PyMOL and Discovery Studio Visualizer.

\subsection{Biological Methods}

2.3.1. Cell Culture and Reagents. Vero cells were maintained in Dulbecco's modified Eagle's medium (DMEM) supplemented with $10 \%$ fetal bovine serum, $1 \%$ penicillin, and streptomycin (P/S; GIBCO \#11995-065), and $2 \mathrm{mM} \mathrm{l-glu-}$ tamine (GIBCO \#11500626). Mycophenolate acid (MA) was purchased from Sigma (SIGMA\#M5255).

2.3.2. Virus Preparation. To examine the anti-Zika effect of berberine derivatives, Zika virus MR766 Africa lineage was used. The strains are stored at the Vietnam National Institute of Hygiene and Epidemiology. This virus inoculated Aedes albopictus mosquito cells, clone C6/36, which was cultured in Eagle's Minimum Essential Medium (EMEM) medium containing $10 \%$ fetal bovine serum and $1 \%$ penicillin and streptomycin. The culture was continued for 14 days until the cytopathic impacts were watched. Viral passages were divided into aliquots and kept at $-80^{\circ} \mathrm{C}$ for use in all experiments.
2.3.3. Immunofluorescence Analysis. Vero cells were seeded at $2 \times 10^{4}$ cells/well onto collagen (Sigma, \#C8919) precoated 96-well, black wall, optical bottom microwell plates (\#165305, ThermoNunc) using DMEM (Gibco, \#11995065) media supplemented with 10\% FBS (OmegaSci \#FB12) and P/S (Gibco, \#15140-122). Cells were pretreated with experimental compounds or positive control (mycophenolate acid, MP) in a $1: 3$ serial dilution at 8 concentrations in triplicate for $2 \mathrm{hr}$ in an incubator. $5 \mu \mathrm{L}$ of ZIKV inoculum (MOI 1) was added to each well. The infected cells were incubated for $24 \mathrm{hr}$ after infection followed by $4 \%$ paraformaldehyde fixation and immunofluorescence assay (IFA). Cells were permeabilized using PBS with $0.1 \%$ TritonX100 and blocked with $2.5 \%$ FBS. Primary antibody, 4G2 (pan-flavivirus, anti-envelope antibody), was diluted in $1 \%$ FBS and incubated on cells for 3 hrs. Secondary antibody, goat anti-mouse Alexa647, and the nuclear stain, Hoechst was applied the following hour. Three images per well were obtained using the 10x objective on Axiovert A1 (Carl Zeiss, Germany). Images were analyzed using Zen imaging and ImageJ software. $\mathrm{IC}_{50}$ (effective concentration) and CC50 (cytotoxicity concentration) values were calculated using Prism v7.0 software. The antiviral activity of tested compounds was evaluated by 8-point DRC analysis in triplicate. Experiments were repeated at least two times independently.

\section{Results and Discussion}

3.1. Synthesis and Structural Characterization. By introducing benzyl groups at the $\mathrm{C}-13$ position of $\mathrm{BBR}$, analogues 4 were synthesized. The synthesis of compound $\mathbf{4}$ involved two steps, whereas compound 5 preparation needed an additional step. Treatment of commercially available BBR 1 with acetone in $5.0 \mathrm{M} \mathrm{NaOH}$ solution at $0^{\circ} \mathrm{C}$ gave the key acetylated intermediates 2 . Then, compound 2 was reacted with benzyl halide or heteroaromatic benzyl halide 3 in $\mathrm{CH}_{3} \mathrm{CN}$ solvent in the presence of $\mathrm{NaI}$ catalyst at $80^{\circ} \mathrm{C}$ to yield the desired compound 4 . Oxoberberine derivatives 5 were formed in the reaction between 4 form salt and hot aqueous alkali in air. The structure of BBR derivatives is presented in Table 1.

3.1.1. Compound 2. ${ }^{1} \mathrm{H}$ NMR (DMSO- $\left.\mathrm{d}_{6}, 400 \mathrm{MHz}\right) \delta=2.03$ (s, 3H), 2.26-2.33 (m, 1H), 2.69-2.75 (m, 2H), 3.89-2.96 (m, $1 \mathrm{H}), 3.17-3.29(\mathrm{~m}, 2 \mathrm{H}), 3.75(\mathrm{~s}, 3 \mathrm{H}), 3.76(\mathrm{~s}, 3 \mathrm{H}), 5.18-5.22$ (m, 1H), 5.99-6.00 (m, 3H), 6.70-6.73 (m, 1H), $6.75(\mathrm{~s}, 1 \mathrm{H})$, 6.84-6.87 (m, 1H), $7.25(\mathrm{~s}, 1 \mathrm{H})$. 
TABLE 1: Chemical structure of BBR derivatives.

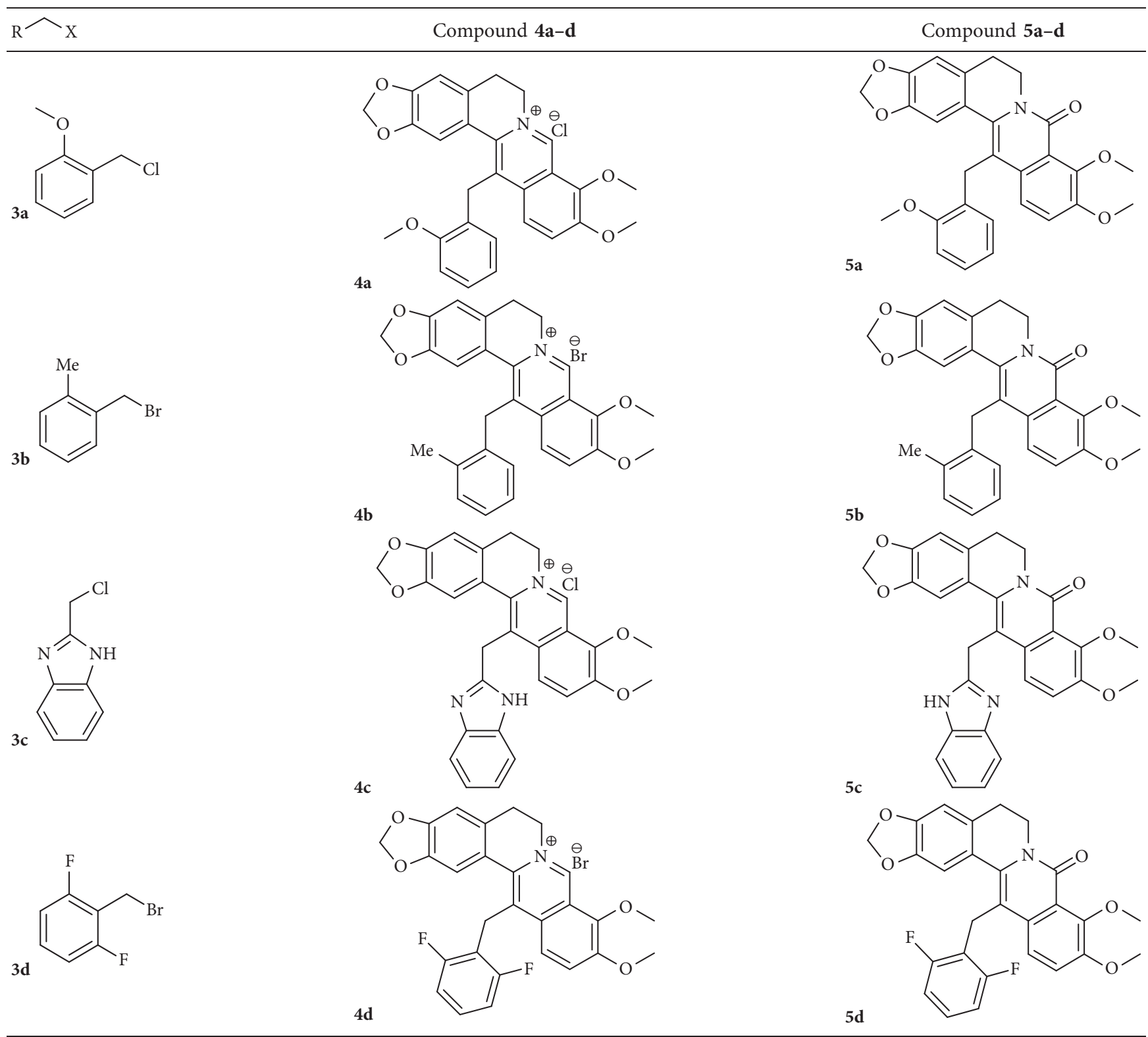

\subsubsection{Compound $\mathbf{4 a - d}$}

4a: ${ }^{1} \mathrm{H} \mathrm{NMR}\left(\mathrm{CDCl}_{3}, 400 \mathrm{MHz}\right) \delta=3.25-3.29(\mathrm{~m}, 2 \mathrm{H})$, $3.95(\mathrm{~s}, 3 \mathrm{H}), 4.01(\mathrm{~s}, 3 \mathrm{H}), 4.40(\mathrm{~s}, 3 \mathrm{H}), 4.51(\mathrm{~s}, 2 \mathrm{H}), 5.21(\mathrm{~s}$, $2 \mathrm{H}), 6.00(\mathrm{~s}, 2 \mathrm{H}), 6.62-6.69(\mathrm{~m}, 2 \mathrm{H}), 6.79-6.93(\mathrm{~m}, 3 \mathrm{H})$, 7.00-7.03 (m, 1H), 7.55-7.72 (m, 2H), $10.44(\mathrm{~s}, 1 \mathrm{H})$

4b: ${ }^{1} \mathrm{H} \mathrm{NMR}\left(\mathrm{CDCl}_{3}, 400 \mathrm{MHz}\right) \delta=2.45(\mathrm{~s}, 3 \mathrm{H}), 3.30-3.34$ (m, 2H), $4.01(\mathrm{~s}, 3 \mathrm{H}), 4.41(\mathrm{~s}, 3 \mathrm{H}), 4.45(\mathrm{~s}, 2 \mathrm{H}), 5.20(\mathrm{~s}$, $2 \mathrm{H}), 6.00(\mathrm{~s}, 2 \mathrm{H}), 6.62-6.67(\mathrm{~m}, 1 \mathrm{H}), 6.82-6.88(\mathrm{~m}, 2 \mathrm{H})$, 7.08-7.11 (m, 1H), 7.21-7.28 (m, 1H), 7.35-7.39 (m, 1H), 7.50-7.55 (m, 1H), 7.69-7.72 (m, 1H), $10.45(\mathrm{~s}, 1 \mathrm{H})$

4c: ${ }^{1} \mathrm{H}$ NMR $\left(\mathrm{CDCl}_{3}, 400 \mathrm{MHz}\right) \delta=3.30(\mathrm{~s}, 2 \mathrm{H}), 3.79$ (s, $3 \mathrm{H}), 4.13(\mathrm{~s}, 3 \mathrm{H}), 4.86(\mathrm{~s}, 2 \mathrm{H}), 5.10(\mathrm{~s}, 2 \mathrm{H}), 6.00(\mathrm{~s}, 2 \mathrm{H}), 6.82$ (s, 1H), 7.17-7.20 (m, 2H), 7.47-7.50 (m, 1H), 7.59-7.62 (m, 2H), $7.71(\mathrm{~s}, 1 \mathrm{H}), 7.79-7.82(\mathrm{~m}, 1 \mathrm{H}), 9.82(\mathrm{~s}, 1 \mathrm{H})$ 4d: ${ }^{1} \mathrm{H}$ NMR $\left(\mathrm{CDCl}_{3}, 400 \mathrm{MHz}\right) \delta=3.23-3.29(\mathrm{~m}, 2 \mathrm{H})$, $4.03(\mathrm{~s}, 3 \mathrm{H}), 4.36(\mathrm{~s}, 3 \mathrm{H}), 4.75(\mathrm{~s}, 2 \mathrm{H}), 5.22(\mathrm{~s}, 2 \mathrm{H}), 6.06$ (s, 2H), 6.82-6.89 (m, 2H), $6.92(\mathrm{~s}, 1 \mathrm{H}), 7.04(\mathrm{~s}, 1 \mathrm{H})$, 7.16-7.24 (m, 1H), $7.74(\mathrm{~s}, 2 \mathrm{H}), 10.44(\mathrm{~s}, 1 \mathrm{H})$

\subsubsection{Compound $5 \boldsymbol{a}-\boldsymbol{d}$}

5a: ${ }^{1} \mathrm{H}$ NMR $\left(\mathrm{CDCl}_{3}, 400 \mathrm{MHz}\right) \delta=2.80-2.84(\mathrm{~m}, 2 \mathrm{H})$, 3.90 (s, 3H), 3.95 (s, 3H), $4.02(\mathrm{~s}, 3 \mathrm{H}), 4.20$ (s, 2H), 5.89 (s, 2H), $6.72(\mathrm{~s}, 1 \mathrm{H}), 6.80-6.90(\mathrm{~m}, 3 \mathrm{H}), 6.94-6.97$ (m, $1 \mathrm{H}), 7.16-7.29(\mathrm{~m}, 3 \mathrm{H})$

5b: ${ }^{1} \mathrm{H} \mathrm{NMR}\left(\mathrm{CDCl}_{3}, 400 \mathrm{MHz}\right) \delta=2.39(\mathrm{~s}, 3 \mathrm{H})$, 2.80-2.84 (m, 2H), $3.90(\mathrm{~s}, 3 \mathrm{H}), 4.05(\mathrm{~s}, 3 \mathrm{H}), 4.16$ (s, $2 \mathrm{H}), 5.90(\mathrm{~s}, 2 \mathrm{H}), 6.43(\mathrm{~s}, 1 \mathrm{H}), 6.77(\mathrm{~s}, 1 \mathrm{H})$, 


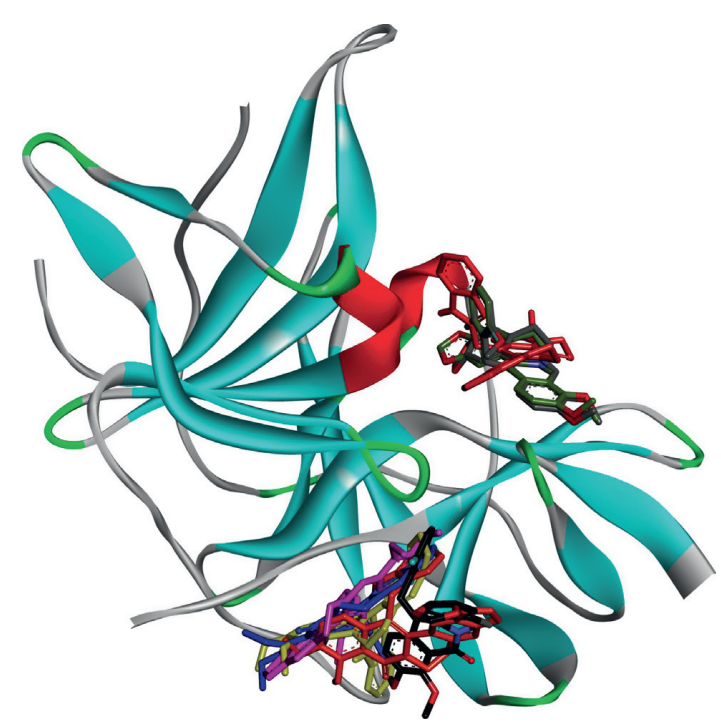

Figure 2: Dock poses of studied compounds (4a: grey; $\mathbf{4 b}$ : violet; $4 \mathrm{c}$ : yellow; $\mathbf{4 d}$ : green; $\mathbf{5 a}$ : orange; $\mathbf{5 b}$ : blue; $\mathbf{5 c}$ : red; $\mathbf{5 d}$ : black) on the binding site of NS2B-NS3 protease of ZIKV.

TABLE 2: Docking results of studied compounds and hydrogen bond interacting residues.

\begin{tabular}{|c|c|c|c|}
\hline \multirow{2}{*}{ Compound } & \multicolumn{3}{|c|}{ ZIKV NS2B-NS3 protein } \\
\hline & Binding energy (kcal/mol) & Interacting residues & $\operatorname{RMSD}(\AA)$ \\
\hline $4 \mathrm{a}$ & -7.18 & His51, Phe84, Ala132, Tyr161 & 1.800 \\
\hline $4 b$ & -6.51 & Ala88, Ile123, Thr166, Gln167, Lys169 & 1.702 \\
\hline $4 c$ & -7.29 & Ala88, Ile123 & 1.821 \\
\hline 4d & -7.31 & His51, Phe84, Ala132, Ser135, Gly151, Gly153, Tyr161 & 1.866 \\
\hline $5 \mathbf{a}$ & -6.09 & Leu78, Asp120, Ile123, Ala164, Ile165, Gly168 & 1.442 \\
\hline 5b & -7.65 & Asp86, Ala88, Ile123, Ala164, Ile165, Thr166 & 1.718 \\
\hline $5 \mathrm{c}$ & -6.97 & His51, Asp129, Ala132, Ser135, Tyr161 & 1.367 \\
\hline 5d & -6.67 & Lys73, Leu78, Thr118, Ile123 & 0.871 \\
\hline BBR & -6.17 & Ala132, Ser135, Tyr150, Gly151, Val155, Gly159, Tyr161 & 1.108 \\
\hline
\end{tabular}

6.89-6.93 $(\mathrm{m}, 1 \mathrm{H}), 7.034-7.19(\mathrm{~m}, 4 \mathrm{H}), 7.25-7.31$ $(\mathrm{m}, 1 \mathrm{H})$

5c: ${ }^{1} \mathrm{H}$ NMR $\left(\mathrm{CDCl}_{3}, 400 \mathrm{MHz}\right) \delta=2.79-2.84(\mathrm{~m}, 2 \mathrm{H})$, $3.79(\mathrm{~s}, 3 \mathrm{H}), 3.86(\mathrm{~s}, 3 \mathrm{H}), 4.21-4.25(\mathrm{~m}, 2 \mathrm{H}), 4.46(\mathrm{~s}$, $2 \mathrm{H}), 5.89(\mathrm{~s}, 2 \mathrm{H}), 6.76(\mathrm{~s}, 1 \mathrm{H}), 7.10-7.40(\mathrm{~m}, 6 \mathrm{H})$, 7.71-7.75 (m, 1H)

5d: ${ }^{1} \mathrm{H} \mathrm{NMR}\left(\mathrm{CDCl}_{3}, 400 \mathrm{MHz}\right) \delta=2.84(\mathrm{~s}, 2 \mathrm{H}), 3.90$ (s, $3 \mathrm{H}), 4.00(\mathrm{~s}, 3 \mathrm{H}), 4.23(\mathrm{~s}, 2 \mathrm{H}), 4.43(\mathrm{~s}, 2 \mathrm{H}), 6.74-6.80$ $(\mathrm{m}, 3 \mathrm{H}), 6.97(\mathrm{~s}, 1 \mathrm{H}), 7.07-7.14(\mathrm{~m}, 1 \mathrm{H}), 7.22-7.25(\mathrm{~m}$, $1 \mathrm{H}), 7.34-7.39(\mathrm{~m}, 1 \mathrm{H})$

3.2. Docking Study. Zika virus NS2B-NS3 protease plays a key role in viral replication; therefore, it is considered as an important antiviral drug target. Several drugs serving as inhibitors of Zika virus NS2B-NS3 have been reported, including bromocriptine with $\mathrm{IC}_{50}$ of $21.6 \mu \mathrm{M}$, novobiocin with $\mathrm{IC}_{50}$ of $14.2 \mu \mathrm{M}$, temoporfin with $\mathrm{IC}_{50}$ of $1.1 \mu \mathrm{M}$, and erythrosine $\mathrm{B}$ with $\mathrm{IC}_{50}$ of $1.7 \mu \mathrm{M}$ [22-24]. To facilitate the development of protease inhibitors with improved potency, proteolytic stability, selectivity, and bioavailability, many strategies have been used to screen for the desired inhibitors. Many studies have exploited in silico model to analyze the interaction between inhibitors and enzymes to find the best promising compounds. For example, Choudhry et al. selected seven potential compounds as antiviral drug candidates with NS2B-NS3 protease as target based on their high GOLD fitness score, high AutoDock Vina score, and $X$-Score binding energy and analyzed the strength of molecular interactions between the active site amino acids and the selected compounds [25]. Another approach is to make chemical modifications of known inhibitors to get higher inhibitory activity. Voss and Nitsche modified the structures of peptidomimetic inhibitors by adding new chemical groups such as benzylamide structure (bZiPro) to form a complex of a P1-P4 segment and different P1 residues [26]. The $\mathrm{IC}_{50}$ of the modified inhibitors were significantly improved compared to the original compound. The new inhibitors may cause synergistic effects between active site and allosteric inhibitors.

A combination of in silico analysis and chemical modification of the target compounds seems to be the best choice for finding inhibitors of Zika virus NS2B-NS3 protease. This is considered as an effective strategy. Using this approach, the antihistaminic chlorcyclizine was discovered as a good inhibitor candidate of Zika virus NS2B-NS3 protease [27]. 
6

Journal of Chemistry

$4 a$
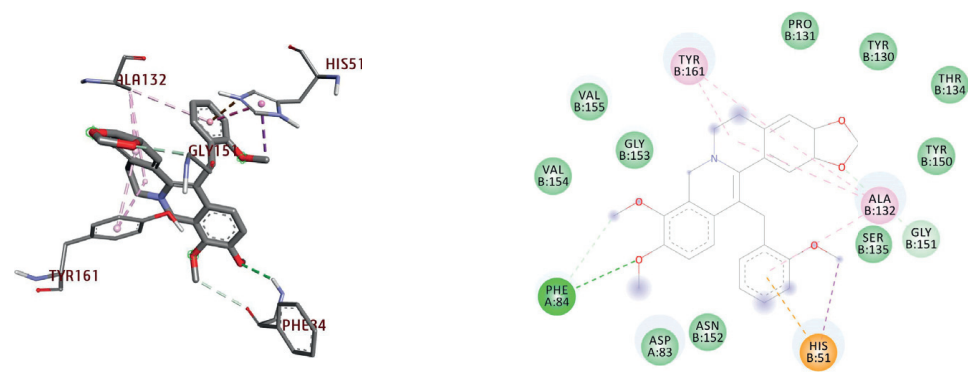

$4 b$
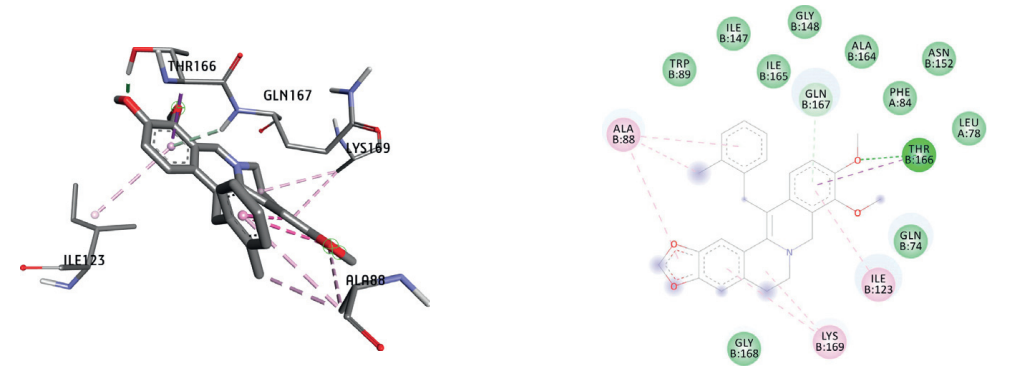

$4 c$
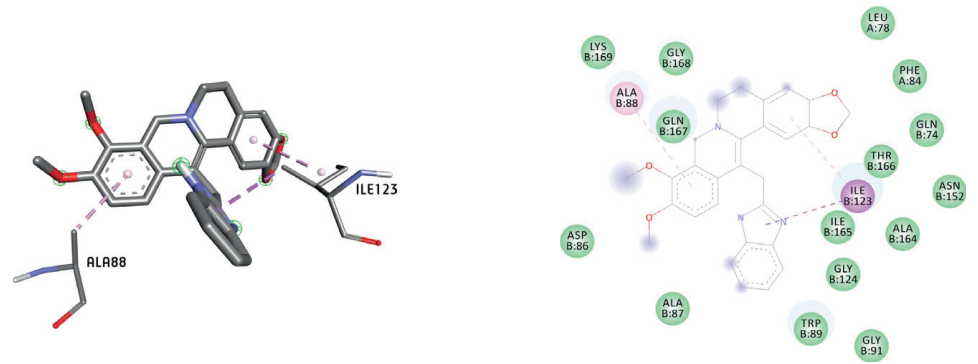

$4 d$
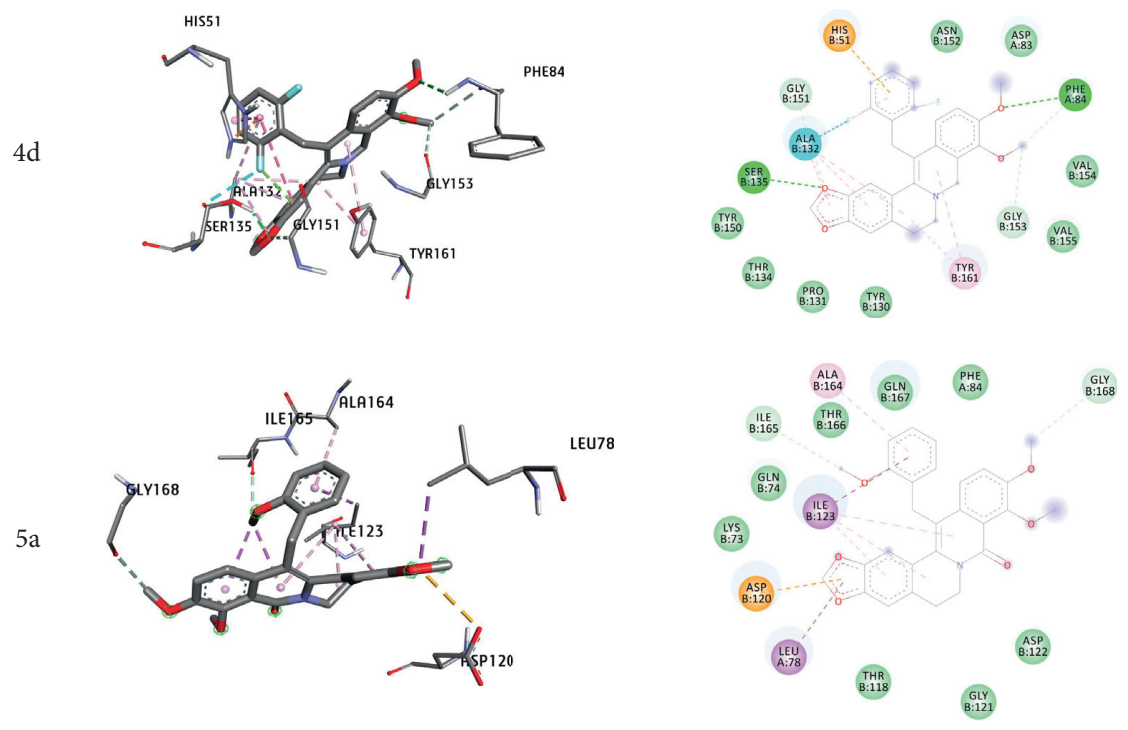

(a)

FIgUre 3: Continued. 

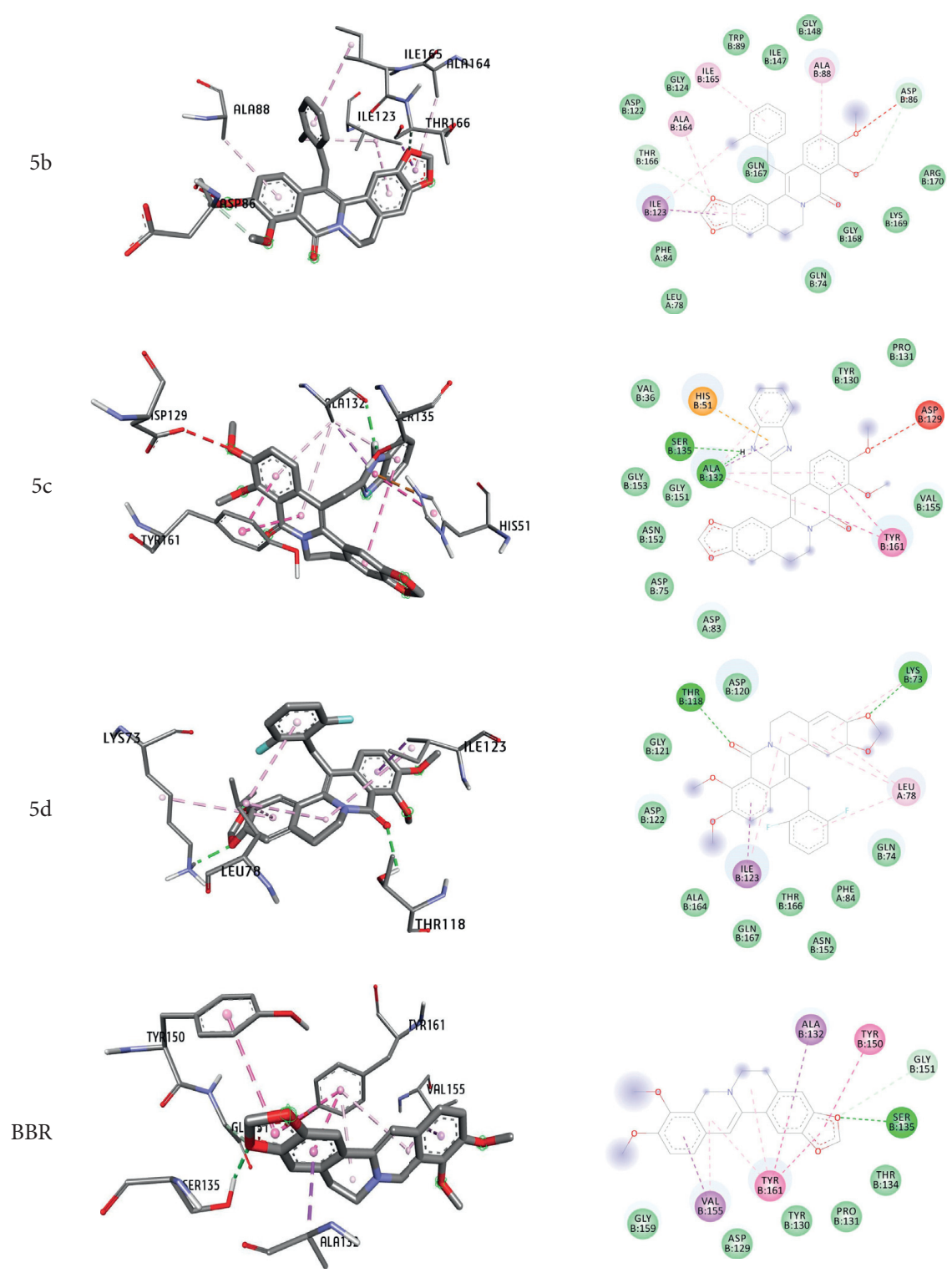

Pi-sigma

Pi-pi T-shaped

Alkyl

Pi-alkyl

Conventional hydrogen bond

(b)

FIgURE 3: Interaction of studied compounds in the NS2B-NS3 binding site suggested by molecular docking studies.

This compound had a grid score of $-24.8 \mathrm{kcal} / \mathrm{mol}$, exhibited the most promising interaction with NS2B-NS3 protease in comparison to crystallography ligand $(-15.6 \mathrm{kcal} / \mathrm{mol})$ by interaction with Tyr161 by hydrophobic interactions in the binding site of NS2B-NS3. Tyr161 is recognized as an important amino acid in substrate molecular recognition. Cytotoxicity and global antiviral activity assay in Vero cells by MTT method showed that chlorcyclizine reduced the ZIKV induced cytopathic effect $\left(\mathrm{IC}_{50}\right.$ of $69.0 \pm 7.3 \mu \mathrm{M}$ and
$S I=1.9$ ), and explicit molecular dynamics simulations implemented on a NAMD program indicated great stability of chlorcyclizine in protease binding site, suggesting the repurposing of chlorcyclizine as a promising finding in antiZIKV drug development.

In this study, molecular docking is utilized to calculate binding affinity and predict binding pose of studied compounds in the active site of ZIKV which is important to investigate the potential mechanism of inhibition activity. 

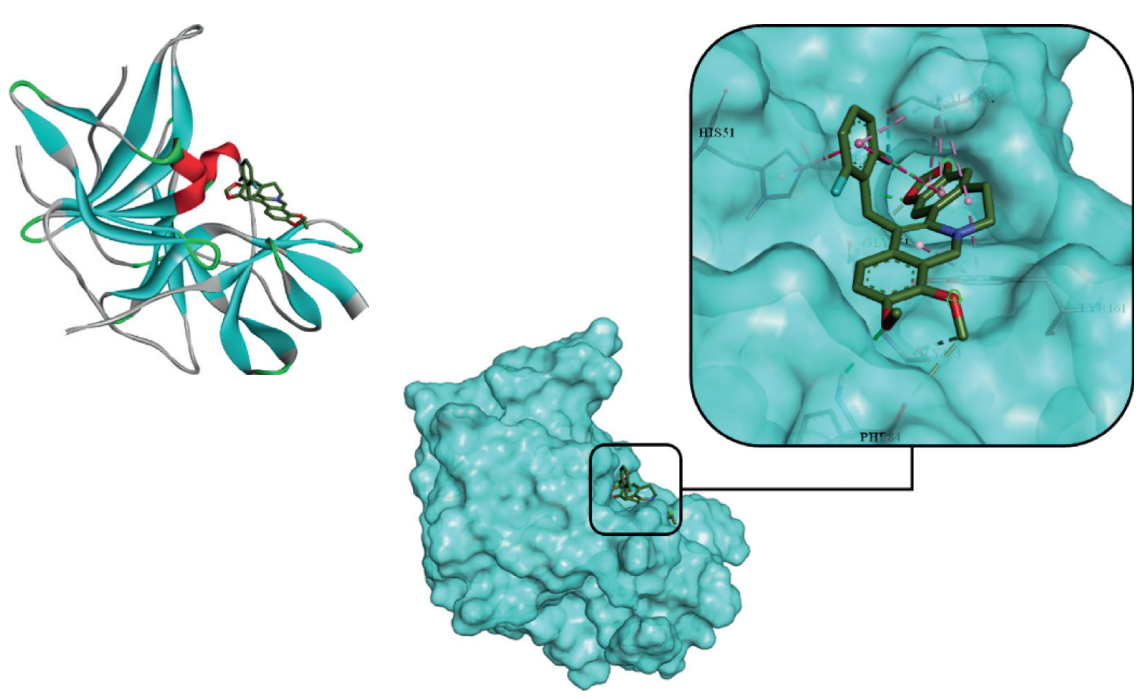

(a)

(b)

FIGURE 4: (a) Compound 4d (thick green lines) in the binding site of NS2B-NS3 protease of ZIKA (ribbon representation). (b) Surface model of the best dock pose of compound 4d with NS2B-NS3 protease.

According to the ranking criteria of AutoDock, the more the negative value of the docking score, the better the binding affinity of the compound towards the targeted receptor [28]. Figure 2 and Table 2 present dock poses of eight ligands in combination with the root-mean-square deviation (RMSD). The docking result is considered reliable when the RMSD value does not exceed $2.0 \AA$. It is reported by Gohlke et al. that ligand calculated partial charge with the PM6 method has been shown to greatly increase docking accuracy and cluster population of the most accurate docking [29]. In 2017, Singh et al. reported that berberine could be a novel inhibitor for NS3-protein preventing from ZIKV; thus, it was selected as a reference ligand for the docking simulation [30].

Of all the docked results, compound $\mathbf{5 b}$ exhibited the most negative dock score $(-7.65 \mathrm{kcal} / \mathrm{mol})$ towards NS2BNS3 which suggest it has the highest binding affinity to this protease; meanwhile, the reference inhibitor (BBR) was recorded with the binding free energy of $-6.17 \mathrm{kcal} / \mathrm{mol}$. Three other derivatives whose docking energies are close to compound $\mathbf{5 b}$ are $\mathbf{4 d}, \mathbf{4 c}$, and $\mathbf{4 a}(-7.31,-7.29$, and $-7.18 \mathrm{kcal} / \mathrm{mol}$, resp.).

BBR binding mode analysis with targeted protease reported that Ala132, Tyr150, Gly151, Val155, Gly159, and Tyr161 are key residues that participated in hydrophobic interactions and Ser135 formed H-bond with this reference ligand. In 2007, Sahoo et al. proved hydrogen bonding interaction between Ser135 and 1,3-dioxolo ring of BBR moiety plays a vital role in the inhibition activity of the compound [20].

Binding mode of the BBR derivatives with compounds 5a-d is displayed in Figure 3. The hydrophobic pockets formed with compound $\mathbf{5}$ a are revealed in Figure 3 involving residues Leu78, Asp120, Ile123, and Ala164. The interaction is further strengthened through two conventional hydrogen bonds with Ile165 and Gly168. Binding orientation analysis of compound $\mathbf{5 b}$, which exhibit the highest binding affinity towards NS2B-NS3 protease, indicated Ala88, Ile123, Ala164, and Ile165 were the main residues involved in weak interactions; meanwhile, Asp86 and Thr166 bonded with compound for two H-bonds. It should be noted that all the residues that participated in interacting with $\mathbf{5 b}$ are not recorded as an important constituent of the active site of targeted protease; therefore, compound $\mathbf{5 b}$ is not considered as "hit" for further evaluation. An array of the hydrophobic interaction was observed as contributed by His51, Asp129, Ala132, and Tyr161 to the binding with compound 5c. The -NH group of compound $\mathbf{5 c}$ at benzimidazole ring forms a hydrogen bond with hydroxyl $(-\mathrm{OH})$ of Ser135.

BBR derivatives $\mathbf{4 a - d}$ were observed to bind effectively with the targeted protease except for compound $\mathbf{4 b}$. Binding orientation analysis exhibited His51, Ala132, and Tyr161 of NS2B-NS3 initiating the hydrophobic interaction with compound $\mathbf{4 a}$ and is further stabilized through a hydrogen bond with Phe84. The noncovalent bonds formed between the binding site of NS2B-NS3 and compound $\mathbf{4 b}$ including Ala88, Ile123, Lys169, and Gln167, and the interaction was further stabilized with one $\mathrm{H}$-bond with Thr166. In the dock pose of compound 4c, Ala88 and Ile123 were the key residues involved in hydrophobic interactions and there were none H-bond formed. Compound $\mathbf{4 d}$ was ranked second in terms of binding affinity. It was observed to form two H-bonding with Phe84 and Ser135; on the other hand, His51, Ala132, Gly151, Gly153, and Tyr161 were the key residues involved in hydrophobic interactions (Figures 3 and 4). Particularly, the 1,3-dioxolo ring of 4d formed hydrogen bond with the hydroxyl (-OH) group of Ser135 which is similar to the reference inhibitor BBR and most of the residues involved in the interaction are pivotal for the function of NS2B-NS3 protease. In conclusion, combining both docking score and binding mode analysis criteria, compound $\mathbf{4 d}$ could be hypothesized as the most potential candidate to inhibit the function of NS2B-NS3 protease. 
TABLE 3: Anti-ZIKV activity of berberines derivatives.

\begin{tabular}{lccc}
\hline Name & $\mathrm{IC}_{50}(\mu \mathrm{M})^{(\mathrm{a})}$ & $\mathrm{CC}_{50}(\mu \mathrm{M})^{(\mathrm{b})}$ & $\mathrm{SI}(\mu \mathrm{M})^{(\mathrm{c})}$ \\
\hline BBR & $15.5 \pm 3.1$ & $62.8 \pm 4.9$ & $\mathbf{4 . 1}$ \\
4a & $35.2 \pm 7.1$ & $112.5 \pm 11.6$ & $\mathbf{3 . 2}$ \\
$\mathbf{4 b}$ & $45.1 \pm 9.6$ & $122.8 \pm 2.1$ & $\mathbf{2 . 7}$ \\
4c & $5.9 \pm 2.2$ & $44 \pm 5.2$ & $\mathbf{7 . 5}$ \\
4d & $\mathbf{5 . 3} \pm \mathbf{1 . 9}$ & $\mathbf{8 0 . 9} \pm \mathbf{5 . 7}$ & $\mathbf{1 5 . 3}$ \\
5a & $37.6 \pm 3.8$ & $90.2 \pm 8.5$ & $\mathbf{2 . 4}$ \\
5b & $9.8 \pm 1.4$ & $25.2 \pm 7.5$ & $\mathbf{2 . 6}$ \\
5c & $116.3 \pm 12.3$ & $325.5 \pm 3.6$ & $\mathbf{2 . 8}$ \\
5d & $29.5 \pm 2.3$ & $79.7 \pm 12.3$ & $\mathbf{2 . 7}$ \\
MA & $0.13 \pm 0.12$ & $>10$ & $>\mathbf{7 6 . 9}$ \\
\hline
\end{tabular}

${ }^{(\mathrm{a})} \mathrm{IC}_{50}$ : half-maximal inhibitory concentration. ${ }^{\mathrm{b})} \mathrm{CC} 50$ values: half-maximal cytotoxicity concentration. ${ }^{(\mathrm{c})} \mathrm{SI}=\mathrm{CC} 50 / \mathrm{EC} 50$ : selective index.

3.3. Evaluation of Anti-ZIKV Activity. Vero cells were treated with berberine, Mycophenolic acid (MA), a positive control, or vehicle $(0.25 \%$ DMSO) for $2 \mathrm{~h}$ before infection with the African ZIKV strain MR766 with a multiplicity of infection (MOI) of 1 . The percentage of infected cells was averaged about $60 \%$ in DMSO condition while it dropped less than $1 \%$ in presence of MA as a positive control. Screening results are summarized in Table 3. Four compounds (BBR, 4a, 4c, 4d) showed activity against ZIKV with a selectivity index $(\mathrm{SI})>3$. Of these, compound $\mathbf{4 d}$ exhibited the highest activity with $\mathrm{IC}_{50}$ at low micromolar levels $(5.3 \mu \mathrm{M})$ and with a high SI $>15,3.7$-fold higher than that of BBR. Five derivatives (4b and $\mathbf{5 a} \mathbf{a}-\mathbf{d})$ showed fewer activities with the SI values more than 2 .

\section{Conclusion}

In our study, BBR which is FDA-approved drug to be effective against flaviviral dengue virus was selected for improving the bioavailability of $\mathrm{BBR}$, consequently enhancing its inhibitory activity against Zika virus NS2B-NS3 protease. The C-13 position of BBR was introduced with benzyl groups and followed by converting to respective 8-oxoberberine derivatives, eight BBR derivatives were synthesized, then molecular docking study and experimental assays were conducted for evaluating their anti-ZIKV activity. The obtained results from the computational part suggested compound $\mathbf{4 d}$ as a potential NS2B-NS3 inhibitor and shed the light on the possible binding mode of BBR and its derivatives with this protein on the basis of molecular docking. BBR binding mode analysis with targeted protease reported that Ala132, Tyr150, Gly151, Val155, Gly159, and Tyr161 are key residues in enzyme active site and hydrogen bonding interaction between Ser135 and 1,3-dioxolo ring of BBR moiety plays a vital role in the inhibition activity of the compound (Sahoo et al.). Here, we synthesized a series of BBR derivatives by introducing the benzyl groups to the $\mathrm{C}-13$ position of $\mathrm{BBR}$ and converting to respective 8Oxoberberine derivatives to generate eight BBR derivatives. Molecular docking study and experimental assays were conducted for evaluating their anti-ZIKV activity of the derivatives. Docking analysis indicated compound $\mathbf{4 d}$ as a potential NS2B-NS3 inhibitor. It interacts with key residues
His51, Phe84, Ala132, Ser135, Gly151, and Gly153 in the binding site of NS2B-NS3 protein, especially Tyr161, a key amino acid in the active site of the enzyme. Our data also suggested that hydrogen bonding with Ser135 might also play an important role in the inhibition mechanism. In agreement with the docking data, the cell-based assay also confirmed 4d exhibited the most potent inhibitory effect against ZIKV infection with $\mathrm{IC}_{50}$ value of $5.3 \pm 1.9$ and SI value of 15.3 , significantly improved compared to the original BBR. Thus, compound $\mathbf{4 d}$ could be served as the most potential candidate to inhibit the function of NS2BNS3 protease. In summary, our results, the synthesize process resulted in 13-substituted BBRs derivatives the compound 4d highlights that the (2,6-difluoro)-benzyl substitution pattern at the $\mathrm{C}-13$ of $\mathrm{BBR}$ is the primary determinant for accomplishing successful noncompetitive inhibition. More works on in vitro and in vivo models are needed to confirm its therapeutic applications.

\section{Data Availability}

All the data used to support the finding of this study are included within the article. Any other data are available from the corresponding author upon request.

\section{Additional Points}

(i) Introduction of heteroaromatic moieties bonded to the 13-position for additional noncovalent aromatic interactions on the cellular target site. (ii) Compound $4 \mathbf{d}$ exhibited the potential NS2B-NS3 protease inhibition activity with a high binding affinity (dock score of $-7.31 \mathrm{kcal} / \mathrm{mol}$ ) and interaction with key residues within the protease active site were observed. (iii) Berberine derivative 4d showed the highest anti-ZIKA virus activity with 3.7-fold SI higher than that of berberine.

\section{Conflicts of Interest}

The authors declare no conflicts of interest.

\section{Acknowledgments}

This research was funded by the Ministry of Education and Training under grant number. B2019-TCT-37.

\section{References}

[1] J. F. W. Chan, G. K. Y. Choi, C. C. Y. Yip, V. C. C. Cheng, and K.-Y. Yuen, "Zika fever and congenital Zika syndrome: an unexpected emerging arboviral disease," Journal of Infection, vol. 72, no. 5, pp. 507-524, 2016.

[2] R. S. Lanciotti, O. L. Kosoy, J. J. Laven et al., "Genetic and serologic properties of zika virus associated with an epidemic, Yap state, Micronesia, 2007," Emerging Infectious Diseases, vol. 14, pp. 1232-1239, 2008.

[3] World Health Organization, WHO Director-General Summarizes the Outcome of the Emergency Committee Regarding Clusters of Microcephaly and Guillain-Barré Syndrome, World Health Organization, Geneva, Switzerland, 2016. 
[4] A. Kumar, Ekavali, K. Chopra, M. Mukherjee, R. Pottabathini, and D. K. Dhull, "Current knowledge and pharmacological profile of berberine: an update," European Journal of Pharmacology, vol. 761, pp. 288-297, 2015.

[5] Y. Zhang, X. Zhang, Y. Zhao et al., "Structural changes of gut microbiota during berberine-mediated prevention of obesity and insulin resistance in high-fat diet-fed rat," PLoS One, vol. 7, 2012.

[6] D. Shum, J. L. Smith, A. J. Hirsch et al., "High-content assay to identify inhibitors of dengue virus infection," Assay and Drug Development Technologies, vol. 8, no. 5, pp. 553-570, 2010.

[7] Y. Ding, X. Ye, J. Zhu, X. Zhu, X. Li, and B. Chen, "Structural modification of berberine alkaloid and their hypoglycemic activity," Journal of Functional Foods, vol. 7, pp. 229-237, 2014.

[8] X. Bian, L. He, and G. Yang, "Synthesis and antihyperglycemic evaluation of various protoberberine derivatives," Bioorganic \& Medicinal Chemistry Letters, vol. 16, no. 5, pp. 1380-1383, 2006.

[9] Y. Zhang, X. Li, D. Zou et al., "Treatment of type 2 diabetes and dyslipidemia with the natural plant alkaloid berberine," The Journal of Clinical Endocrinology \& Metabolism, vol. 93, no. 7, pp. 2559-2565, 2008.

[10] H. Ruan, Y. Y. Zhan, J. Hou et al., "Berberine binds RXR $\alpha$ to suppress $\beta$-catenin signaling in colon cancer cells," Oncogene, vol. 36 , no. 50 , pp. $6906-6918,2017$.

[11] R. Gautam and S. M. Jachak, "Recent developments in antiinflammatory natural products," Medicinal Research Reviews, vol. 29, no. 5, pp. 767-820, 2009.

[12] M. Bahar, Y. Deng, X. Zhu et al., "Potent antiprotozoal activity of a novel semi-synthetic berberine derivative," Bioorganic \& Medicinal Chemistry Letters, vol. 21, no. 9, pp. 2606-2610, 2011.

[13] K. D. Park, J. H. Lee, S. H. Kim, T. H. Kang, J. S. Moon, and S. U. Kim, "Synthesis of 13-(substituted benzyl) berberine and berberrubine derivatives as antifungal agents," Bioorganic \& Medicinal Chemistry Letters, vol. 16, no. 15, pp. 3913-3916, 2006.

[14] Y. X. Wang, W. Q. Pang, Q. X. Zeng et al., "Synthesis and biological evaluation of new berberine derivatives as cancer immunotherapy agents through targeting IDO1," European Journal of Medicinal Chemistry, vol. 143, pp. 1858-1868, 2018.

[15] L. Wang, H. Kong, M. Jin et al., "Synthesis of disaccharide modified berberine derivatives and their anti-diabetic investigation in zebrafish using a fluorescence-based technology," Organic \& Biomolecular Chemistry, vol. 18, no. 18, pp. 3563-3574, 2020.

[16] D. Bhowmik, M. Hossain, F. Buzzetti, R. D. Auria, P. Lombardi, and G. Suresh Kumar, "Biophysical studies on the effect of the 13 position substitution of the anticancer alkaloid berberine on Its DNA binding," The Journal of Physical Chemistry B, vol. 116, pp. 2314-2324, 2012.

[17] L. Zhang, J. Li, F. Ma et al., "Synthesis and cytotoxicity evaluation of 13-n-alkyl berberine and palmatine analogues as anticancer agents," Molecules, vol. 17, no. 10, pp. 11294-11302, 2012.

[18] Z. Bikadi and E. Hazai, "Application of the PM6 semi-empirical method to modeling proteins enhances docking accuracy of autodock," Journal of Cheminformatics, vol. 1, 2009.

[19] P. M. M. Araújo, L. P. da Silva, and J. C. G. Esteves da Silva, "Theoretical analysis of the binding of potential inhibitors to protein kinases MK2 and MK3," Medicinal Chemistry, vol. 11, no. 6, pp. 573-579, 2015.
[20] M. Sahoo, L. Jena, S. Daf, and S. Kumar, "Virtual screening for potential inhibitors of NS3 protein of zika virus," Genomics \& Informatics, vol. 14, 2016.

[21] J. A. Kang, S. Kim, M. Park et al., "Ciclopirox inhibits Hepatitis B virus secretion by blocking capsid assembly," Nature Communications, vol. 10, 2019.

[22] J. F.-W. Chan, K. K.-H. Chik, S. Yuan et al., "Novel antiviral activity and mechanism of bromocriptine as a Zika virus NS2B-NS3 protease inhibitor," Antiviral Research, vol. 141, pp. 29-37, 2017.

[23] Z. Li, S. Sakamuru, R. Huang et al., "Erythrosin B is a potent and broad-spectrum orthosteric inhibitor of the flavivirus NS2B-NS3 protease," Antiviral Research, vol. 150, pp. 217225, 2018.

[24] L. Wang, R. Liang, Y. Gao et al., "Development of smallmolecule inhibitors against zika virus infection," Frontiers in Microbiology, vol. 10, 2019.

[25] H. Choudhry, F. A. Alzahrani, M. A. Hassan et al., "Zika virus targeting by screening inhibitors against NS2B/NS3 protease," BioMed Research International, vol. 2019, Article ID 3947245, 11 pages, 2019.

[26] S. Voss and C. Nitsche, "Inhibitors of the Zika virus protease NS2B-NS3," Bioorganic \& Medicinal Chemistry Letters, vol. 30, 2020.

[27] F. R. S. Santos, D. A. F. Nunes, W. G. Lima et al., "Identification of zika virus NS2B-NS3 protease inhibitors by structure-based virtual screening and drug repurposing approaches," Journal of Chemical Information and Modeling, vol. 60, no. 2, pp. 731-737, 2020.

[28] N. T. Nguyen, T. H. Nguyen, T. N. H. Pham et al., "Autodock vina adopts more accurate binding poses but autodock 4 forms better binding affinity," Journal of Chemical Information and Modeling, vol. 60, no. 1, pp. 204-211, 2019.

[29] H. Gohlke, M. Hendlich, and G. Klebe, "Knowledge-based scoring function to predict protein-ligand interactions," Journal of Molecular Biology, vol. 295, no. 2, pp. 337-356, 2000.

[30] A. P. Singh, A. Kumar, and V. Srivastava, "In silico molecular docking of antiviral drugs against NS3 potential of Zika virus," International Journal of Scientific and Innovative Research, vol. 5, pp. 37-40, 2017. 